\title{
ORGANIZACIÓN POR PROCESOS Y SU INFLUENCIA EN EL SERVICIO AL CLIENTE DE LA EMPRESA INVERSIONES PINTO S.A.C
} ORGANIZATION BY PROCESS AND ITS INFLUENCE ON THE CUSTOMER SERVICE OF
THE COMPANY INVERSIONES PINTO S.A.C

Thalía E. Franco Valverde, Ingeniero ${ }^{1}$, Isabel F. Rojo Montoro, Ingeniero ${ }^{1}$, Elizabeth K. Bravo Huivin, Magister ${ }^{1}$, Cesia E. Boñon Silva, Magister ${ }^{1}$, Nelson A. Angeles Quiñones, Magister ${ }^{1}$, and Juan M. Deza Castillo, Magister ${ }^{1}$

\footnotetext{
${ }^{1}$ Universidad Privada del Norte, Perú, thaliafrancov@gmail.com, isarojom7@gmail.com, kristina.bravo@upn.pe, cesia.bonon@upn.edu.pe,nelson.angeles@upn.edu.pe,juan.deza@upn.edu.pe
}

\begin{abstract}
Resumen- La presente investigación busca determinar la influencia de una organización por procesos en el servicio al cliente de la empresa Inversiones Pinto S.A.C. La investigación según el propósito es aplicada y según el diseño es preexperimental. Para la toma de información se emplearon distintos instrumentos, como: guías de entrevista para trabajadores y gerente, fichas de observación, check list en el área de ventas y cuestionarios para los clientes. Respecto a la metodología empleada, se inició por la determinación de la situación en la que se encontraba la empresa; donde se evidenció que su forma de gestión afectaba la eficiencia de sus procesos y el uso óptimo de los recursos disponibles. De acuerdo con ello, se planteó un nuevo modelo de gestión y organización de los procesos, para posteriormente efectuar su implementación y, por último, evaluar los resultados para determinar de qué forma influenció en el nivel de servicio de la empresa. Como resultado se obtuvo que, la propuesta influyó positivamente en el servicio al cliente, ya que antes de ello la experiencia de compra llegaba a una sumatoria de 199; mientras que una vez hecha la implementación se elevó a 251. Asimismo, las ventas aumentaron en un $2.9 \%$ y el resultado neto también incrementó en un 1.79\%. Lo mismo sucedió, en el plano ambiental, a través del plan de reciclaje se logró concientizar y fomentar la reutilización y separación de los distintos desechos que genera la empresa. Con ello, se logró reducir en un $42 \%$ el uso de papel principalmente. Demostrándose la viabilidad de la propuesta en ambos sentidos.

Palabras Clave: Organización por procesos, servicio al cliente.
\end{abstract}

Abstract-This research seeks to determine the influence of an organization by processes in the customer service of the company Inversiones Pinto S.A.C. The work methodology according to the purpose is applied and according to the design it is pre-experimental. Different instruments were used to collect information, such as: interview guides for workers and managers, observation files, a check list in the sales area, and questionnaires for customers. Regarding the development of the investigation, it began by determining the situation in which the company was; where it was evidenced that its form of management affected the efficiency of its processes and the optimal use of available resources. Accordingly, a new model of management and organization of the processes was proposed, to later carry out its implementation and, finally, evaluate the results to determine how it influenced the level of service of the company. As a result, it was obtained that the proposal had a positive influence on customer service, since before that the shopping experience reached a sum of 199; while once the implementation was made, it rose to 251. Likewise, sales increased by $2.9 \%$ and the net result also increased by $1.79 \%$. The same happened, on the environmental level, through the recycling plan it was possible to raise awareness and promote the reuse and separation of the different waste generated by the company. With this, it was possible to reduce the use of paper by $42 \%$ mainly. Demonstrating the viability of the proposal in both directions.

Keywords: Organization by processes, customer service.

\section{INTRODUCIÓN}

En la actualidad, las organizaciones del sector automotriz juegan un papel muy importante como propulsor en el desarrollo de otros sectores de valor agregado, provocando que varios países tengan la iniciativa de apostar en el desarrollo y fortalecimiento de este rubro. Además, este sector siempre se ha caracterizado por su constante innovación tecnológica en todos los ámbitos. Tal es así, que se ha venido apostando por el uso de energías y tecnologías más limpias. Según [1]; en China, Shenzen se ha convertido en la primera ciudad a nivel global en sustituir toda su flota de autobuses diésel del transporte público por buses eléctricos. Actualmente, la ciudad cuenta con más de 16.000 autobuses eléctricos. Este es un caso de éxito que se puede replicar. Si bien, Latinoamérica ya inició esa transición, el camino aún es largo.

Siguiendo el contexto latinoamericano, de acuerdo con [2], el sector automotor obtuvo un buen año, alcanzando ventas por 5'886,120 unidades de vehículos, de los cuales el primer

\footnotetext{
Digital Object Identifier (DOI):

http://dx.doi.org/10.18687/LEIRD2021.1.1.29

ISSN: 2414-6390 ISBN: 978-958-52071-9-6
} 
trimestre se comercializó 1.39 millones de unidades de vehículos.

En el ámbito nacional, el panorama del sector va tomando velocidad y se muestra positivo, alcanzando la venta de 15,367 unidades en enero. Así, con un avance de $3.8 \%$, el mercado intenta dejar atrás siete meses de caídas consecutivas, según la Asociación Automotriz del Perú (AAP)". [3]. Por otro lado, [4] dice: Con un crecimiento de $2,4 \%$, el sector automotor en el Perú cerró el 2019 con el mejor desempeño dentro de los países de la Alianza del Pacífico, así lo informó la Asociación Automotriz del Perú (AAP) al tiempo de señalar que en segundo lugar se lo lleva Colombia con un crecimiento de 1,7\%, mientras que México y Chile reportaron contracciones de $-7,7 \%$ y $-10,6 \%$; respectivamente.

Así como va incrementando la demanda, aumenta también la oferta, es por ello por lo que el entorno empresarial actual es cada vez más exigente. Por consecuente para sobrevivir se debe mantener una mejora constante de los negocios, y por ende de sus procesos. [5]. Por lo tanto, para [6] la gestión en base a procesos es una forma para que la empresa pueda conseguir eficaz y eficientemente sus objetivos. Para ello, los procesos deben ser parte de un sistema integral que permita el logro de resultados globales en la empresa, teniendo que estar alineados a los objetivos y estos, a la misma vez, podrán estar relacionados a uno o varios grupos de interés en la empresa.

Por otro lado, el estudio realizado por [7] nos informa que el modelo de gestión de procesos crea el marco organizativo ideal para ejecutar la optimización de gastos, los planes de mejora continua y beneficio de recursos. Para esto, es imprescindible que para la toma de decisiones se analice el proceso de forma integral, pensando en el bien de la empresa.

Además, el mejoramiento continuo son pequeños y permanentes perfeccionamientos de un sistema, proceso o unidad organizacional dentro de la empresa. La mejora continua de procesos productivos o administrativos para obtener productos y servicios flexibles, adaptables, de calidad y económicos es una meta propuesta para toda empresa. [8]

Para precaver en su organización, la declinación y pérdida de eficacia de la cultura y prácticas del servicio, proyecte el servicio al cliente como una estrategia dinámica. Su plan del servicio también debe ser dinámico. Debe serlo porque las tendencias surgen y desaparecen, por ende, los comportamientos sociales y económicos varían. [9]

Conjuntamente, sin cliente no hay servicio y solo este sabe los factores clave utilizará para evaluarnos. Las perspectivas del cliente son la base principal de una organización que busca la excelencia en su servicio. [10]. Por lo tanto, según [11] un buen servicio al cliente puede llegar a ser un elemento promocional tanto para las ventas tan poderosas como para los descuentos, la publicidad o la venta personal.

Cada proceso dentro de una organización debe elaborarse bajo el supuesto de satisfacer una necesidad del cliente, luego con los recursos existentes de manera eficiente y eficaz los colaboradores tienen la responsabilidad de asegurar la ejecución de estos y trabajar en renovarlos continuamente. [12]. Por otro lado, la competitividad de las organizaciones está cada vez más asociada a la eficiencia de los procesos y actividades. La gestión de los procesos de una organización de forma adecuada satisface los requerimientos del cliente, y de manera eficaz, con el menor costo posible lleva al éxito y competitividad. Es por ello por lo que las organizaciones deben enfocarse en Sistemas de Gestión que prioricen la estructura de estas por medio de los procesos. [13]

El servicio al cliente siempre ha ido durante el tiempo mejorando, cada empresa tiene sus estrategias para atraer a sus consumidores potenciales y fidelizarlos. Por consiguiente; Kotler, [14] dicen que en donde existan diferentes tipos de personas con deseos, percepciones, preferencias y criterios de compra totalmente diferencias e individuales. El competidor inteligente debe diseñar y entregar ofertas bien definidas para los mercados objetivos.

De esta manera, se han observado e identificado que los clientes en la actualidad son más delicados al servicio que llegan a recibir de sus abastecedores, por la competencia existente en los mercados y la variedad de estrategias que aplican las empresas para la satisfacción de su pública objetivo, para ello buscan la mejor opción en tiempo, dinero y servicio. [13]

En general, la cultura del servicio es parte importante de la organización, el cliente viene a ser el corazón del modelo organizacional y al plantear un cambio en la estructura de la organización, veremos que en lo más alto de la pirámide se encuentra el cliente, seguido de las divisiones, subgerencias y en la base la gerencia quien debe tramitar la relación con los consumidores. [15]. A ello, se suma la opinión del profesor Carlos Rodríguez, especialista en estrategia, que indica que todas las empresas sin importar su sector o tamaño deben mantenerse alertas a la globalización para una evolución favorable en el mercado. Un ejemplo claro, de la fusión de ambas ideas, es lo que hizo McDonald's en su establecimiento del aeropuerto de Frankfurt, donde decidió equipar a sus trabajadores con dispositivos móviles para tomar las ordenes, dinamizando los tiempos de espera y ofrecer un mejor servicio. [16]

El secreto para una gestión por procesos tenga éxito es tener una visión clara que esté relacionada con los objetivos clave del negocio y los productos de suma importancia para los clientes. En lugar de basar los proyectos de mejora de procesos en la estructura existente, es fundamental entender y comprender cuáles son las capacidades necesarias para entregar los mejores resultados al cliente y luego determinar la mejor estructura para ello. [17]

La empresa Inversiones Pinto S.A.C. nació de la necesidad de los consumidores por satisfacer sus necesidades en cuanto a repuestos eléctricos automotrices. Conforme ha ido creciendo la empresa, la exigencia de los clientes ha ido aumentando y los problemas se han ido presentando. Una de las debilidades principales es su forma de gestión, no se aprovechan todos los recursos que se tiene a disposición y que le aportarían un mayor 
nivel de competitividad en el mercado. En base a ello, se busca organizar la empresa por procesos que permitan potenciar el beneficio de todos los recursos que posee y que aporten en el logro de los objetivos; asimismo modificar o eliminar aquellos que no ofrezcan una contribución eficiente.

\section{OBJETIVOS}

\section{A. Objetivo General}

Determinar la influencia de una organización por procesos en el servicio al cliente de la empresa Inversiones Pinto S.A.C., 2018.

\section{B. Objetivos Específicos}

- Establecer la situación actual del servicio al cliente de la empresa inversiones Pinto S.A.C.

- Implementar la organización por procesos en la empresa Inversiones Pinto S.A.C.

- Evaluar el servicio al cliente posterior a la implementación de la organización por procesos.

- Evaluar económica, social y ambientalmente la organización por procesos.

\section{MATERIAL Y MÉTODOS}

Según, [18] en su Manual de Metodología de la Investigación, la presente investigación es Aplicada, Experimental - Preexperimental, indican que en "las muestras no probabilísticas, la elección de los elementos no depende de la probabilidad, sino de causas relacionadas con las características de la investigación o propósitos del investigador.

La población son los clientes de la empresa Inversiones Pinto S.A.C. y la muestra será aleatoriamente constituida por 30 personas de sexo masculino entre 18 y 65 años.

Para la recolección de información se utilizaron instrumentos como: fichas de observación, check list en el área de ventas, guías de entrevista, tanto para los trabajadores como para el gerente general; con el fin de obtener toda la información posible acerca de los procesos, tal como: funciones de los trabajadores, fortalezas y debilidades de la empresa. Así mismo, también se efectuaron cuestionarios a los clientes para determinar su nivel de satisfacción.(Tabla1)

TABLA 1.

\section{TECNICA E INSTRUMENTOS}

\begin{tabular}{|c|l|l|}
\hline TÉCNICA & $\begin{array}{c}\text { INSTRUM } \\
\text { ENTO }\end{array}$ & \multicolumn{1}{c|}{ LOGRO } \\
\hline Observación & $\begin{array}{l}\text { Fichas de } \\
\text { observación }\end{array}$ & $\begin{array}{l}\text { Este instrumento, permitirá que se tenga un registro de lo } \\
\text { que sucede realmente con cada uno de los procesos } \\
\text { estudiados, teniendo en cuenta las horas de inicio y fin de } \\
\text { los procesos observados, con el fin de obtener los tiempos } \\
\text { promedio para cada proceso. También se podrá visualizar } \\
\text { prácticas o conductas que muchas veces no son relevantes } \\
\text { para los participantes del proceso, pero que si son } \\
\text { percibidas por los observadores. }\end{array}$ \\
\hline Observación & Check list & $\begin{array}{l}\text { El check list cuenta con doce preguntas y un apartado de } \\
\text { observaciones, en el que el inspector o entrevistador podrá } \\
\text { anotar cosas relevantes del desarrollo del proceso } \\
\text { evaluado. Este instrumento permitirá comprobar de manera } \\
\text { sistemática y ordenada el cumplimiento de aspectos } \\
\text { importantes relacionados al área de ventas }\end{array}$ \\
\hline Entrevista & $\begin{array}{l}\text { El tercer y cuarto instrumento son las guías de entrevista } \\
\text { base, que serán utilizados para los trabajadores y gerente } \\
\text { general. La guía de entrevista del gerente general; contará } \\
\text { con diecisiete preguntas acerca de las ventas, costos, }\end{array}$ \\
\hline
\end{tabular}

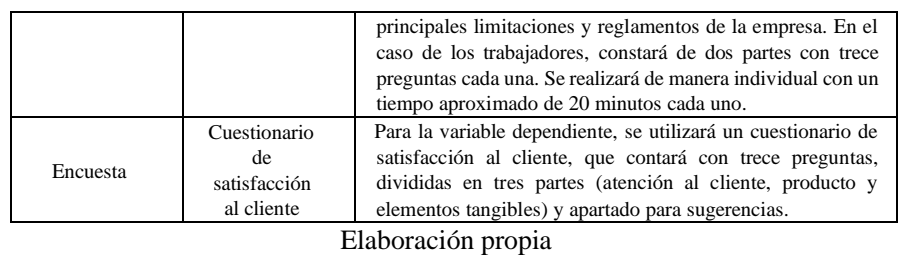

\section{METODOLOGÍA PARA LA PROPUESTA}

Los investigadores desarrollaron la propuesta en 3 etapas las cuales consta de: Análisis externo, Análisis interno y Perfil estratégico propuesto.

La Tabla 2 se aprecia dichas etapas con las principales actividades.

TABLA 2

ETAPAS DE LA PROPUESTA

\begin{tabular}{|c|c|c|}
\hline ITEM & ETAPAS & SUBETAPAS \\
\hline 1 & $\begin{array}{c}\text { Etapa 1: } \\
\text { Análisis externo }\end{array}$ & $\begin{array}{l}\text { - Madriz de perfil competitivo (MPC) } \\
\text { - Modelo de las Cinco Fuerzas de Porter } \\
\text { - Madriz de Evaluación de Factores Externos } \\
\text { (EFE) }\end{array}$ \\
\hline 2 & $\begin{array}{c}\text { Etapa 2: } \\
\text { Análisis interno }\end{array}$ & $\begin{array}{l}\text { - Matriz de Evaluación de Factores Internos } \\
\text { - Matriz FODA }\end{array}$ \\
\hline 3 & $\begin{array}{c}\text { Etapa 3: Perfil } \\
\text { estratégico } \\
\text { propuesto }\end{array}$ & $\begin{array}{l}\text { - Determinación de la disciplina de valor } \\
\checkmark \text { Definición de la cadena de valor } \\
\checkmark \text { Estructura de los procesos } \\
\checkmark \text { Diagramas de procesos } \\
\text { - Procesos Estratégicos } \\
\text { - Procesos Operativos } \\
\text { - Procesos de Soporte } \\
\text { - Estructura de Organigrama Propuesto } \\
\text { - Descripción del Organigrama } \\
\text { Propuesto } \\
\text { - Manual de organización y funciones (MOF) }\end{array}$ \\
\hline
\end{tabular}

\section{A. Etapa 1: Análisis Externo}

\section{Matriz de perfil competitivo (MPC)}

La Figura 1 muestra la matriz de perfil competitivo, que identifica a los principales competidores de la empresa, mediante la evaluación de factores críticos de éxito en la industria. Asimismo, se evidencia las fortalezas y debilidades en contraposición de los competidores. Como se puede observar el competidor más fuerte es Resedisa con un valor ponderado de 3.32 .

\begin{tabular}{|c|c|c|c|c|c|c|c|c|c|}
\hline \multirow{2}{*}{ Factores criticos de éxito } & \multirow{2}{*}{ Peso } & \multicolumn{2}{|c|}{ RESEDISA } & \multicolumn{2}{|c|}{ RESEA } & \multicolumn{2}{|c|}{ MB REPUESTOS } & \multicolumn{2}{|c|}{ INVERSIONES PINTO } \\
\hline & & Clasificación & Peso Ponderado & Clasificación & Peso Ponderado & Clasificación & Peso Ponderado & Clasificación & Peso Ponderado \\
\hline Calidad del producto & 0.15 & 4 & 0.6 & 4 & 0.6 & 4 & 0.6 & 4 & 0.6 \\
\hline Servicio al cliente & 0.15 & 3 & 0.45 & 3 & 0.45 & 3 & 0.45 & 3 & 0.45 \\
\hline Publicidad & 0.05 & 4 & 0.2 & 2 & 0.1 & 1 & 0.05 & 2 & 0.1 \\
\hline $\begin{array}{c}\text { Tecenologias de } \\
\text { intormación }\end{array}$ & 0.09 & 3 & 0.27 & 3 & 0.27 & 3 & 0.27 & 2 & 0.18 \\
\hline Precios & 0.2 & 3 & 0.6 & 3 & 0.6 & 4 & 0.8 & 4 & 0.8 \\
\hline Infraestructura & 0.08 & 4 & 0.32 & 2 & 0.16 & 1 & 0.08 & 3 & 0.24 \\
\hline Promociones & 0.06 & 3 & 0.18 & 2 & 0.12 & 1 & 0.06 & 2 & 0.12 \\
\hline Variedad de productos & 0.08 & 3 & 0.24 & 3 & 0.24 & 3 & 0.24 & 3 & 0.24 \\
\hline Fidelización & 0.1 & 3 & 0.3 & 3 & 0.3 & 2 & 0.2 & 3 & 0.3 \\
\hline Reputación de la marca & 0.04 & 4 & 0.16 & 4 & 0.16 & 4 & 0.16 & 4 & 0.16 \\
\hline Total & 1 & & 3.32 & & 3 & & 2.91 & & 3.19 \\
\hline
\end{tabular}

Fig.1. Matriz de perfil competitivo. Elaboración propia

$1^{\text {sh }}$ LACCEI International Multiconference on Entrepreneurship, Innovation and Regional Development - LEIRD 2021: "Ideas to Overcome and Emerge from the Pandemic Crisis", Virtual Edition, December 9-10, 2021. 
La clasificación de la calificación en la matriz de perfil competitivo es: $4=$ Gran fortaleza, $3=$ Fuerza menor, $2=$ Debilidad menor y $1=$ Gran debilidad

\section{Modelo de las Cinco Fuerzas de Porter}

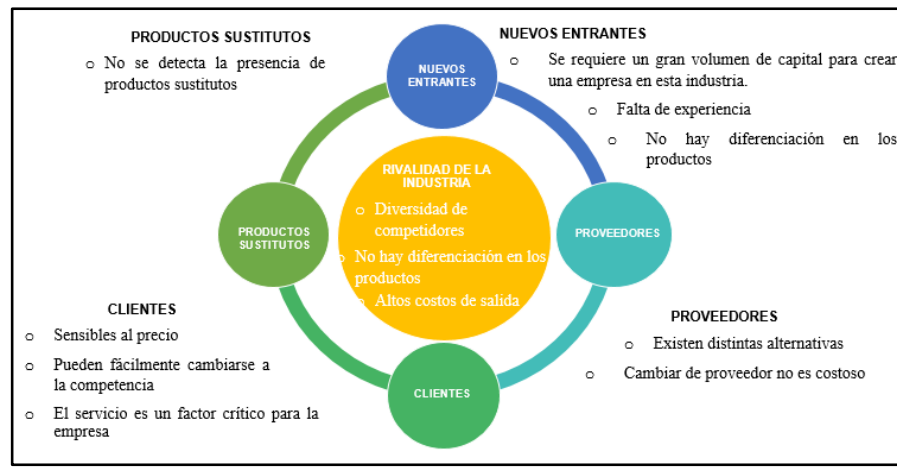

Fig.2. Matriz de Porter.

Elaboración propia

En la Figura 2, una de las barreras de entrada principales de esta industria, es la gran inversión que se necesita para poner en marcha una empresa de repuestos eléctricos, principalmente para la adquisición de inventario. Otro factor importante que puede hacer desistir a los posibles nuevos entrantes es la falta de experiencia y conocimiento de los productos, ya que para ingresar es de vital importancia tener conocimientos básicos sobre mecánica automotriz. Por otro lado, no existe diferenciación en los productos que se comercializan en esta industria, lo que significa una desventaja para los nuevos entrantes, debido a que tendrían que competir contra empresas que ya están establecidas; y es que estas cuentan ya con una marca reconocida y clientes fidelizados, lo que obliga a los nuevos entrantes a realizar importantes inversiones en publicidad o ingresar con precios muy bajos. Por lo tanto, la amenaza de nuevos entrantes es baja, debido a que las barreras de entrada que presenta la industria son muy fuertes.

El poder de negociación de los proveedores es bajo, ya que existe variedad de oferta para adquirir los repuestos eléctricos, así mismo, no es costoso cambiar de proveedor, por lo tanto, no es conveniente para ellos incrementar los precios o cambiar las condiciones de compra.

Matriz de Evaluación de Factores ExternoS (EFE)
\begin{tabular}{|l|c|c|c|}
\hline OPORTUNIDADEs & PESO & CALFICACIÓN & PONDERADO \\
\hline Crecimiento económico & 0.08 & 2 & 0.16 \\
\hline Alianzas estratégicas & 0.09 & 3 & 0.27 \\
\hline Disponibiridad de Tl para mejorar el desarrollo de los procesos & 0.09 & 2 & 0.18 \\
\hline Publicidad en redes sociales & 0.07 & 2 & 0.14 \\
\hline Fuentes de financiamiento externo & 0.05 & 2 & 0.1 \\
\hline Eajo poder de negociación de los proveedores & 0.05 & 3 & 0.15 \\
\hline Inexistencia de productos sustitutos & 0.09 & 4 & 0.36 \\
\hline sUB TOTAL & & & 1.36 \\
\hline AMENAZAS & & & \\
\hline AUmento de la competencia & 0.09 & 3 & 0.27 \\
\hline Competidores con precios más económicos & 0.09 & 3 & 0.27 \\
\hline
\end{tabular}

\begin{tabular}{|l|c|c|c|}
\hline Inestabilidad política del país & 0.05 & 2 & 0.1 \\
\hline Regulaciones más exigentes & 0.02 & 3 & 0.06 \\
\hline Desastres naturales & 0.05 & 2 & 0.1 \\
\hline Descenso de la demanda & 0.09 & 2 & 0.18 \\
\hline Alta rivalidad entre competidores & 0.08 & 2 & 0.16 \\
\hline Incremento de los costos de transporte & 0.01 & 3 & 0.03 \\
\hline SUB TOTAL & & & 1.17 \\
\hline TOTAL & & & 2.53 \\
\hline
\end{tabular}

Fig.3. Matriz de EFE.

Elaboración propia

La Figura 3 muestra la matriz de evaluación de factores externos, en la que se analiza las oportunidades y amenazas que afronta la empresa. Se utiliza el valor del ponderado total de las oportunidades para contrastarlo con el ponderado total de las amenazas. En este caso el ponderado total de las oportunidades supera el ponderado total de las amenazas, siendo 1.36 y 1.17 respectivamente, por lo tanto, se deduce que el entorno en el que se desempeña la empresa es favorable.

\section{B. Etapa 2: Análisis Interno}

\section{Matriz de Evaluación de Factores Internos}

En la Figura 4 se muestra el análisis de las fuerzas y debilidades que afronta la empresa, el uso de esta matriz de factores internos permite realizar una auditoría interna, el total del ponderado de las fortalezas se utiliza para contrastar el ponderado total de las debilidades. En este caso, el ponderado total de las fortalezas supera al pondera total de las debilidades, siendo 3,28 y 1,09 respectivamente, para la empresa de estudio los resultados son favorables en el estudio de sus fuerzas internas siendo el ponderado por encima del 2,5.

\begin{tabular}{|l|c|c|c|}
\hline \multicolumn{1}{|c|}{ FACTORES CLAVES DE ÉXITO } & PESO & CALIFICACIÓN & PONDERADO \\
\hline Variedad de Productos & 0,05 & 3 & 0,15 \\
\hline Precios accesibles & 0,10 & 3 & 0,30 \\
\hline Buen servicio & 0,15 & 4 & 0,60 \\
\hline Ubicación estratégica para atención al público & 0,15 & 4 & 0,45 \\
\hline Entorno laboral favorable & 0,12 & 3 & 0,36 \\
\hline Local propio & 0,13 & 4 & 0,52 \\
\hline Calidad de los productos & 0,10 & 3 & 0,30 \\
\hline Conocimiento y experiencia en el sector & 0,06 & 3 & 0,18 \\
\hline Buena imagen & 0,06 & 3 & 0,18 \\
\hline Amplias instalaciones & 0,08 & 4 & 0,24 \\
\hline & & $5 U B$ TOTAL & 3,28 \\
\hline \multicolumn{1}{|c|}{ DEBILIDADES } & & & \\
\hline Procesos no definidos & 0,13 & 1 & 0,13 \\
\hline Control de inventarios & 0,13 & 1 & 0,13 \\
\hline Capacitación de clientes internos. & 0,08 & 1 & 0,08 \\
\hline Falta de plan estratégico & 0,13 & 1 & 0,13 \\
\hline Promociones de los productos & 0,05 & 2 & 0,10 \\
\hline Inexistente control de los procesos & 0,13 & 1 & 0,13 \\
\hline
\end{tabular}

$1^{\text {sh }}$ LACCEI International Multiconference on Entrepreneurship, Innovation and Regional Development - LEIRD 2021: "Ideas to Overcome and Emerge from the Pandemic Crisis", Virtual Edition, December 9-10, 2021. 


\begin{tabular}{|l|c|c|c|}
\hline $\begin{array}{l}\text { Capacidad tecnológica acorde con las exigencias del } \\
\text { mercado }\end{array}$ & 0,06 & 1 & 0,06 \\
\hline Deficiente presencia en redes sociales & 0,05 & 2 & 0,10 \\
\hline Deficiente publicidad de la empresa & 0,05 & 2 & 0,10 \\
\hline Personal sin experiencia en el rubro & 0,08 & 1 & 0,08 \\
\hline Poca diferenciación de los productos & 0,05 & 1 & 0,05 \\
\hline & & SUB TOTAL & 1,09 \\
\hline & & & $\mathbf{4 , 3 7}$ \\
\hline
\end{tabular}

Fig.4. Matriz de EFI.

Elaboración propia

\section{Matriz de Fortalezas, Debilidades, Oportunidades y Amenazas (FODA)}

En la Figura 5 se consolidan todos los factores identificados en la matriz EFI y EFE, es decir, tanto fortalezas, oportunidades, debilidades y amenazas.

\begin{tabular}{|c|c|}
\hline FORTALEZAS & OPORTUNIDADES \\
\hline $\begin{array}{l}\text { - Variedad de Productos } \\
\text { - Precios accesibles } \\
\text { - Ubicación estratégica para atención al público } \\
\text { - Entorno laboral favorable } \\
\text { - Local propio } \\
\text { - Calidad de los productos } \\
\text { - Conocimiento y experiencia en el sector } \\
\text { - Buena imagen } \\
\text { - Amplias instalaciones }\end{array}$ & $\begin{array}{l}\text { - Crecimiento económico } \\
\text { - Alianzas estratégicas } \\
\text { - Disponibilidad de TI para mejorar el desarrollo } \\
\text { de los procesos } \\
\text { - Publicidad en redes sociales } \\
\text { - Fuentes de financiamiento externo } \\
\text { - Bajo poder de negociación de los proveedores } \\
\text { - Inexistencia de productos sustitutos }\end{array}$ \\
\hline DEBILIDADES & AMENAZAS \\
\hline $\begin{array}{l}\text { - Procesos no definidos } \\
\text { - Control de inventarios } \\
\text { - Capacitación de clientes internos. } \\
\text { - Falta de plan estratégico } \\
\text { - Promociones de los productos } \\
\text { - Inexistente control de los procesos } \\
\text { - Capacidad tecnológica acorde con las exigencias } \\
\text { - del mercado } \\
\text { - Deficiente presencia en redes sociales } \\
\text { - Personal sin experiencia en el rubro } \\
\text { - Poca diferenciación de los productos }\end{array}$ & $\begin{array}{l}\text { - Aumento de la competencia } \\
\text { - Competidores con precios más económicos } \\
\text { - Inestabilidad política del país } \\
\text { - Regulaciones más exigentes } \\
\text { - Desastres naturales } \\
\text { - Descenso de la demanda } \\
\text { - Alta rivalidad entre competidores } \\
\text { - Incremento de los costos de transporte }\end{array}$ \\
\hline
\end{tabular}

Fig.5. Matriz de FODA.

Elaboración propia

\section{Etapa 3: Perfil estratégico propuesto}

El análisis tanto interno como externo han permitido definir las características de la estructura de organización, que debe ser aplicada en la empresa Inversiones Pinto S.A.C. para conseguir reducir o, en el mejor de los casos, eliminar sus deficiencias. A continuación, se propone una organización que facilite la alineación de los procesos organizacionales con los objetivos estratégicos.

\section{Determinación de la disciplina de valor}

En esta etapa se especifica la estrategia para crear y sostener la ventaja competitiva, por lo tanto, se trabajará con "Disciplinas de Valor. La cadena de valor es una herramienta básica para determinar la ventaja competitiva y encontrar formas de crearla y mantenerla. La empresa define como su disciplina de valor la Excelencia Operativa, que le permitirá $1^{\text {sh }}$ LACCEI International Multiconference on Entrepreneurship, Innovation and Regional Development - LEIRD 2021: "Ideas to Overcome and Emerge from the Pandemic Crisis", Virtual Edition, December 9 - 10, 2021. tener un mayor nivel de eficiencia en sus procesos, por lo tanto, podrá mejorar su capacidad de respuesta ante cualquier eventualidad que se presente en la organización, que influirá directamente en la calidad del servicio.

\section{Definición de la cadena de valor}

La Figura 6 especifica los procesos de la empresa implicados en brindar valor a todos los clientes, con el fin de obtener una ventaja competitiva.

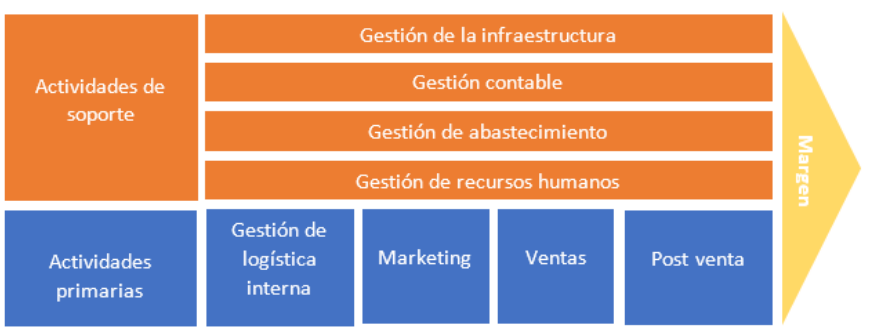

Fig.6. Cadena de Valor Elaboración propia

\section{Estructura de los procesos}

En la Figura 7 se representa de manera gráfica todos los procesos de la empresa Inversiones Pinto S.A.C. tanto proceso de soporte, procesos estratégicos y procesos operativos, en forma interrelacionada.

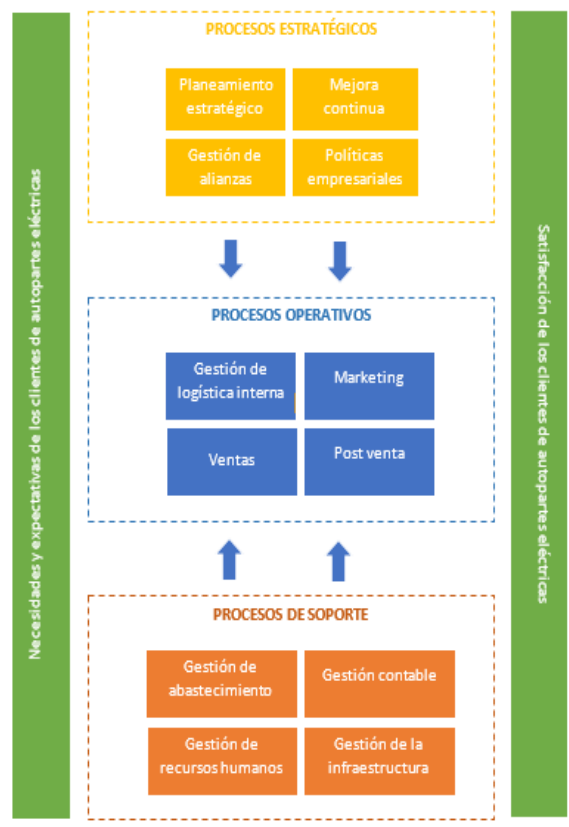

Fig.7. Estructura de los procesos Elaboración propia

\section{Diagramas de procesos}

A continuación, se representará de manera gráfica los procesos por niveles, con las actividades que los conforman y su respectiva secuencia lógica. 


\section{Procesos Operativos}

La organización tiene como procesos operativos la Gestión de logística interna, Marketing, Ventas y Post Ventas. A continuación, se detalla el proceso de Ventas.

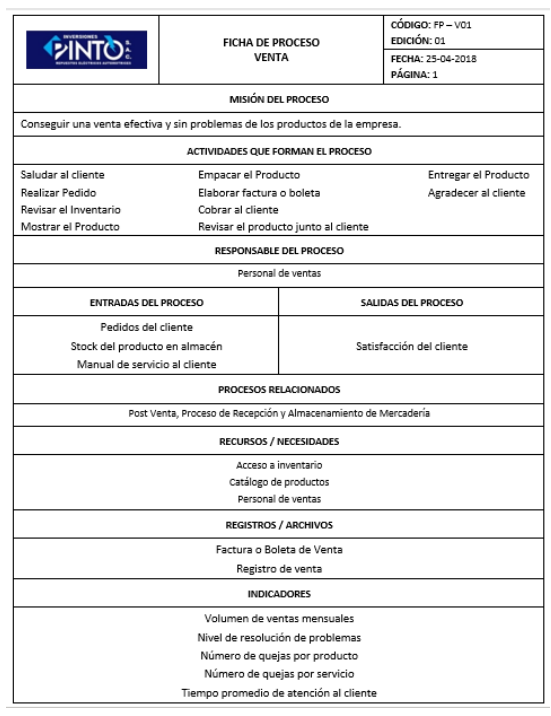

Fig.10. Ficha de procesos Elaboración propia

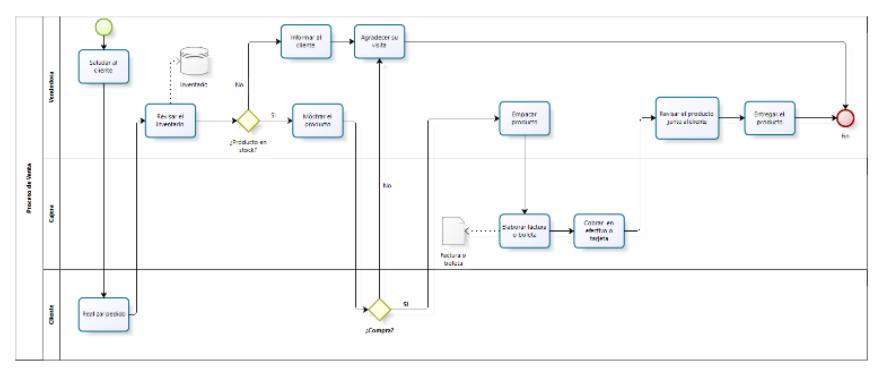

Fig.11. Diagrama de procesos Elaboración propia

\section{Estructura de Organigrama Propuesto}

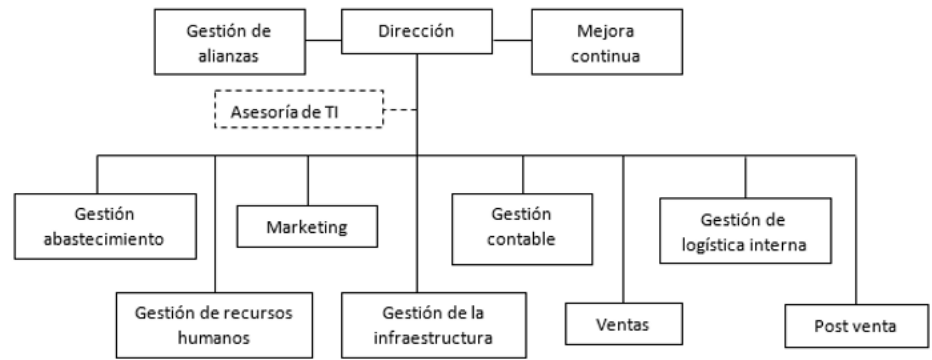

Fig.14. Organigrama

Elaboración propia

La elaboración de la nueva estructura organizacional se rigió a una premisa base, esta es la reducción, en lo posible, de la jerarquización. De esta manera, la comunicación en los trabajadores y directivos será más fluida y directa, ya que no habrá necesidad de intermediarios como en las organizaciones verticales. También permitirá una mayor agilidad en la toma de decisiones y presentación de reportes, debido a la ausencia de filtros continuos para que una decisión sea aceptada, haciendo que la organización sea más ágil y adaptable a los cambios. Es así, que se plantea lo siguiente:

TABLA 3.

PROPUESTAS ORGANIZACIONALES Propuestas organizacionales

$\checkmark$ Crear el proceso de gestión de alianzas, que establezca acciones a realizar y metas a conseguir, mediante un plan con un enfoque adecuado.

$\checkmark$ Crea proceso de mejora continua, que asegure la mayor calidad posible de los productos, servicios y procesos de una empresa.

$\checkmark$ Crear proceso de post venta, que permita el análisis, conclusión, acción y respuesta de los requerimientos o reclamos de los clientes.

$\checkmark$ Crear puesto de asistente administrativo, que planifique y coordine actividades generales. Asimismo, gestione las actividades dentro del proceso de gestión de recursos humanos, ya que el gerente general no se da abasto para el buen desarrollo de dichas actividades.

$\checkmark$ Crear puesto de contador, que se encargue de la presentación de la información financiera para la toma de decisiones y cumplimiento de las obligaciones fiscales.

Elaboración propia

En la Tabla 4 se detalla a modo de ejemplo la descripción del puesto de asistente administrativo de la organización.

TABLA 4

DESCRIPCIÓN DEL PUESTO DE ASISTENTE ADMINISTRATIVO

\begin{tabular}{|l|l}
\hline NOMBRE DEL CARGO & Asistente Administrativo
\end{tabular}

\section{RESUMEN GENERAL DEL CARGO}

Planificar y coordinar actividades generales, cumpliendo con las políticas y procedimientos definidos por la empresa.

\section{FUNCIONES ESPECIFICAS DEL CARGO}

Redactar documentos diversos.

Tramitar los depósitos bancarios y mantener registro de estos.

Archivar y llevar control de los documentos de la empresa.

Realizar reclutamiento y selección de personal.

Planificar programas de capacitación al personal

Evaluar el desempeño del personal.

Realizar pago al personal.

Publicar contenido acerca de los productos y servicios en la página de Facebook.

Responder las preguntas hechas por los clientes mediante la página de Facebook.

Realiza cualquier otra tarea afín que le sea asignada.

\section{AUTORIDAD Y RELACIÓN}

Responde ante la gerencia general de la empresa.

\section{REQUISITOS DEL CARGO}

Egresado en Administración o carreras afines.

Experiencia mínima de 1 año en puestos similares.

Conocimientos básicos en computación e informática.

$$
\text { Elaboración propia }
$$

\section{Plan de Gestión Ambiental}

\section{A) Efectos de la contaminación ambiental}

a. Daños en los estados de la salud

La contaminación puede afectar de distintas maneras nuestra salud, tal es así que puede causar enfermedades respiratorias, enfermedades dermatológicas, enfermedades cardiovasculares, sordera a causa de la contaminación acústica y diversos tipos de cáncer.

b. Desaparición de la capa de ozono

Poco a poco los altos índices de contaminación van provocando su debilitamiento y la aparición de un agujero. 
Causando la proliferación de diversas enfermedades en las personas, así como efectos negativos en la naturaleza. Por ejemplo, reducción de los casquetes polares y aumentos de las temperaturas.

c. Derretimiento de los casquetes polares

Las altas temperaturas generan el deshielo de los casquetes polares, desapareciendo diversas especies propias de esas zonas. Asimismo, se incrementan los niveles del mar, generando la desaparición de pequeñas islas, y la afectación de diferentes regiones marítimas.

\section{B) Visión del plan de gestión ambiental}

Ser la empresa más comprometida e innovadora, en nuestro rubro, respecto al reciclaje a nivel local con la cooperación de los trabajadores aportando ideas creativas para alcanzar el objetivo de cuidar el medio ambiente.

\section{C) Misión del plan de gestión ambiental}

Lograr disminuir el uso excesivo de materiales que estén afectando al planeta, crear conciencia y hábito de cuidado del medio ambiente en todo el personal de la empresa.

\section{D) Política Integral}

Inversiones Pinto S.A.C., empresa dedicada a la venta de autopartes eléctricas, se compromete con el mejoramiento continuo del desempeño del Plan de Gestión Ambiental y a que en sus actividades y nuevos proyectos se incluyan todos los aspectos en materia de preservación del medio ambiente, con el objetivo de lograr la satisfacción interna y de sus clientes. Para esto, la Política Integral se cumple mediante los siguientes compromisos:

o Reducir los impactos socio ambiental generado por actividades propias de la operación, aumentando la preservación del medio ambiente.

- La empresa se compromete a dedicar los recursos humanos y financieros necesarios para la planeación, ejecución y evaluación del plan de gestión ambiental.

- Fomentar la participación y concientización de todos los niveles de la organización sobre el cuidado ambiental.

\section{E) Objetivos}

- Contar con estrategias que permitan dar un uso más eficiente a los recursos.

- Sensibilizar al personal de la empresa, respecto a impacto de la vida cotidiana en el medio ambiente.

\section{F) Estrategias}

Para comenzar a adoptar una cultura del cuidado del medio ambiente, se adoptan las siguientes estrategias y acciones:

- Separar la basura: Colocar los tachos de basura con diferentes colores para ubicar de manera correcta cada residuo según su composición. (E1)
- Apagar las luces: Por más obvio que parezca, son muchas las veces que las luces de un área están encendidas, aunque esta no esté siendo ocupada. (E2)

- Evitar dejar aparatos enchufados: Aunque los aparatos estén apagados siguen consumiendo energía, por eso es importante desenchufarlos. (E3)

- Apagar las PC en caso de recesos de más de 30 minutos y al final de la jornada laboral. (E4)

- Cerrar los caños correctamente: Cuando no se estén utilizando, cerrarlos bien y verificar que no existan fugas. (E5)

- Equipar los caños con boquillas que reduzcan el flujo. (E6) o Utilizar aguas grises (aguas residuales del trapeado de instalaciones, por ejemplo) para el riego de jardines. (E7)

- Utilizar eficientemente el papel: Usar el papel por ambas caras. (E8)

- Fomentar el uso de internet para enviar y/o recibir correos, reportes, publicidad y todos los documentos que sea posible. (E9)

o Dividir el material reutilizable y clasificarlo, para posteriormente mandar los residuos a empresas dedicadas al reprocesamiento. (E10)

- Crear campañas creativas y dinámicas sobre la contaminación y sus efectos. (E11)

\section{G) Proceso De Reciclaje}

En el siguiente diagrama de proceso, se muestran las actividades secuenciales a seguir para la obtención de una nueva materia prima o producto, a partir de materiales ya en desuso.

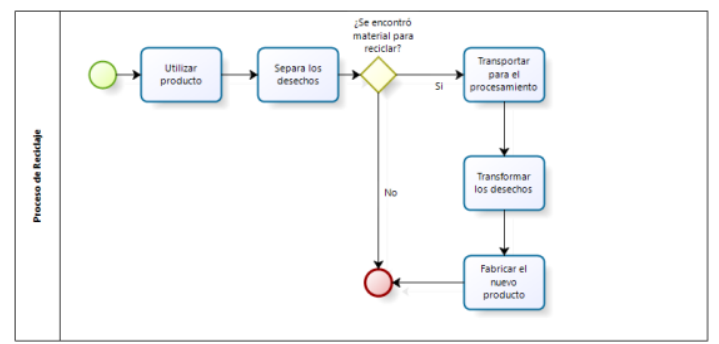

Fig.14. Proceso de reciclaje Elaboración propia

\section{H) Resultados}

\begin{tabular}{|c|c|c|c|c|c|c|c|c|}
\hline INDICADORES & FÓRMULAS & $\begin{array}{l}\text { UNIDAD DE } \\
\text { MEDIDA }\end{array}$ & PERIODICIDAD & ÓPTIMO & TOLERABLE & DEFICIENTE & PRETEST & POSTEST \\
\hline $\begin{array}{l}\text { Consumo de } \\
\text { Agua }\end{array}$ & $\begin{array}{l}\text { Volumen de } \\
\text { consumo de agua }\end{array}$ & s/. & Mensual & $\begin{array}{l}\mathrm{s} / .60 \mathrm{a} \\
\mathrm{s} / .74\end{array}$ & $\begin{array}{l}s / .75 \text { a } \\
\text { S/.89.00 }\end{array}$ & $\begin{array}{c}\mathrm{S} / .90 \mathrm{a} \\
\text { más }\end{array}$ & S/. 93.80 & S/.71.90 \\
\hline $\begin{array}{ll} & \\
\text { Consumo de } & \text { de } \\
\text { Energía } & \\
\text { Eléctrica } & \end{array}$ & $\begin{array}{l}\text { Volumen de } \\
\text { consumo de } \\
\text { energía eléctrica }\end{array}$ & s/. & Mensual & $\begin{array}{l}S / 170 a \\
S / 184\end{array}$ & $\begin{array}{l}\mathrm{S} / 185 \text { a } \\
\mathrm{S} / .199\end{array}$ & $\begin{array}{l}\text { S/ } 200 \text { a } \\
\text { más }\end{array}$ & s/. 196.30 & S/. 187.10 \\
\hline $\begin{array}{l}\text { Consumo de } \\
\text { Papel }\end{array}$ & $\begin{array}{ll}\begin{array}{l}\text { Volumen } \\
\text { consumo }\end{array} & \text { de } \\
\end{array}$ & s/. & Mensual & $\begin{array}{l}\mathrm{S} / 18 \mathrm{a} \\
\mathrm{s} / .23\end{array}$ & S/.24 a s/.35 & $\begin{array}{l}\text { S/ } 36 \text { a } \\
\text { más }\end{array}$ & $s / 45$ & s/18 \\
\hline $\begin{array}{l}\text { Concientización } \\
\text { de Personal }\end{array}$ & $\begin{array}{lr}\text { Número de } \\
\text { charlas realizadas }\end{array}$ & \# & Mensual & 2 & 1 & 0 & 0 & 2 \\
\hline
\end{tabular}

Fig.14. Resultados ambientales Elaboración propia

En la tabla anterior, se visualiza la reducción del uso de recursos, respecto al período previo a la implantación del plan. El consumo del agua se redujo en S/.21.80, el consumo de papel se redujo en $\mathrm{S} / .27$ y se realizaron las dos charlas programadas para la concientización del personal. Esto 
conlleva a que los tres indicadores mencionados anteriormente, se encuentren en un rango óptimo. Por otro lado, el consumo de energía eléctrica se redujo en S/.9.20, manteniéndose en un rango tolerable. Sumándose esta reducción de costos, se obtiene un total de $\mathrm{S} / .58$ soles mensuales, que al año pueden ser S/.696.

Se propusieron distintas estrategias que conllevaron a la reducción del uso de recursos, tales como: la energía eléctrica, agua y papel. Obteniendo un manejo de recursos sostenible en la empresa. Se recomienda, que una vez alcanzados los objetivos propuestos en este plan; se sigan fijando nuevos, con el fin de ser una empresa economicante rentable, así como social y ambientalmente responsable.

Se realizaron dos charlas mensuales, con el fin de concientizar al personal sobre el cuidado ambiente y el efecto que tiene cada una de sus acciones y hábitos, para que no solo manejen buenas prácticas en el trabajo, sino también en su vida cotidiana.

\section{Balance Comparativo}

TABLA 5

BALANCE COMPARATIVO

\begin{tabular}{|c|c|c|c|c|}
\hline \multicolumn{5}{|c|}{$\begin{array}{l}\text { BALANCE COMPARATIVO PARA LOS EJERCICIOS TERMINADOS DEL } 30 \text { DE ABRIL Y } 30 \\
\text { DE JUNIO DEL } 2018\end{array}$} \\
\hline $\begin{array}{l}\text { INVERSIONES PINTO } \\
\text { S.A.C. }\end{array}$ & ABRIL & JUNIO & VARIACIÓN & $\%$ \\
\hline \multicolumn{5}{|l|}{ INGRESOS OPERATIVOS } \\
\hline VENTAS & S/ 94,599.99 & $\mathrm{S} / 97,347.83$ & $\mathrm{~S} / 2,747.84$ & $2.90 \%$ \\
\hline COSTO DE VENTAS & $\mathrm{S} / 74,564.68$ & $\mathrm{~S} / 75,581.50$ & $-\mathrm{S} / 1,016.82$ & $1.33 \%$ \\
\hline RESULTADO BRUTO & S/ $20,035.31$ & $\mathrm{~S} / 21,766.33$ & $\mathrm{~S} / 1,731.02$ & $7.90 \%$ \\
\hline $\begin{array}{lr}\text { GASTOS } & \text { DE } \\
\text { ADMINISTRACIÓN } & \mathrm{Y} \\
\text { VENTAS } & \\
\end{array}$ & & & & \\
\hline SUELDOS & S/ 9,265.60 & S/ $10,479.25$ & $-\mathrm{S} / 1,213.65$ & $-11.50 \%$ \\
\hline LEYES SOCIALES & S/ 4,294.00 & S/ 4,740.79 & $-\mathrm{S} / 446.79$ & $-9.4 \%$ \\
\hline SEGUROS & $\mathrm{S} / 1,115.79$ & $\mathrm{~S} / 1,297.14$ & $-\mathrm{S} / 181.35$ & $-13.9 \%$ \\
\hline ALQUILERES PERDIDOS & $\mathrm{S} /$ & $\mathrm{S} / \quad-$ & $\mathrm{S} /$ & \\
\hline TOTAL GAV & S/ 14,675.39 & S/ 16,517.18 & -S/ $1,841.79$ & $-11.15 \%$ \\
\hline \multicolumn{5}{|l|}{ OTROS INGRESOS } \\
\hline DESCUENTOS OBTENIDOS & $\mathrm{S} / 410.00$ & $\mathrm{~S} / 627.00$ & $\mathrm{~S} / 217.00$ & $34.5 \%$ \\
\hline \multicolumn{5}{|l|}{ GASTOS FINANCIEROS } \\
\hline INTERESES PERDIDOS & $\mathrm{S} / 9.50$ & $\mathrm{~S} / 12.10$ & $-\mathrm{S} / 2.60$ & $-21.5 \%$ \\
\hline GASTOS BANCARIOS & $\mathrm{S} / 78.50$ & $\mathrm{~S} / 78.50$ & $\mathrm{~S} /-$ & $0.0 \%$ \\
\hline RESULTADO NETO & S/ 5,681.92 & S/ $5,785.55$ & S/ 103.63 & $1.79 \%$ \\
\hline
\end{tabular}

Elaboración propia

Respecto a la variabilidad porcentual de los períodos analizados, se evidencia un crecimiento económico de $1.79 \%$. Resultado positivo, teniendo en cuenta que, en Perú, la venta de vehículos livianos y pesados se redujo un $8 \%$ respecto al año 2017, debido a que entró en vigor el Impuesto Selectivo al Consumo para este rubro. [18]

\section{RESULTADOS}

\section{ATENCIÓN AL CLIENTE}

Para la evaluacion de la satisfacción lo conformaron los clientes que realizaron compras en el primer trimestre del año 2020, cuya muestra fue de 30 clientes.
El nivel de satisfacción del cliente en relación con la atención en mostradores pasó de un nivel bueno de $63 \%$ a $87 \%$.

TABLA 6

NIVEL DE SATISFACCIÓN DEL CLIENTE EN RELACION A LA ATENCIÓN EN MOSTRADORES

\begin{tabular}{|c|c|c|c|c|}
\hline \multirow{2}{*}{$\begin{array}{c}\text { DIMENS } \\
\text { IÓN }\end{array}$} & $\begin{array}{c}\text { ATENCIÓN AL } \\
\text { CLIENTE }\end{array}$ & $\begin{array}{c}\text { SUB } \\
\text { DIMENSIÓ } \\
\text { N }\end{array}$ & \multicolumn{2}{|c|}{$\begin{array}{c}\text { ATENCIÓN EN } \\
\text { MOSTRADORES }\end{array}$} \\
\hline \multicolumn{2}{|c|}{ INDICADOR } & CRITERIO & ANTES & DESPUÉS \\
\hline \multirow{4}{*}{$\begin{array}{c}\text { Nivel de satisfacción en los } \\
\text { mostradores }\end{array}$} & Excelente & $0 \%$ & $0 \%$ \\
\cline { 2 - 5 } & Bueno & $63 \%$ & $87 \%$ \\
\cline { 2 - 5 } & Regular & $30 \%$ & $13 \%$ \\
\cline { 2 - 4 } & Malo & $7 \%$ & $0 \%$ \\
\cline { 2 - 4 } & Muy Malo & $0 \%$ & $0 \%$ \\
\hline
\end{tabular}

Elaboración propia

El nivel de satisfacción del cliente en relación con la atención en la caja pasó de un nivel bueno de $54 \%$ a $83 \%$.

TABLA 7

NIVEL DE SATISFACCIÓN DEL CLIENTE EN RELACION A LA ATENCIÓN EN CAJA

\begin{tabular}{|c|c|c|c|c|}
\hline $\begin{array}{c}\text { DIMENSI } \\
\text { ÓN }\end{array}$ & $\begin{array}{c}\text { ATENCIÓN AL } \\
\text { CLIENTE }\end{array}$ & $\begin{array}{c}\text { SUB } \\
\text { DIMENSIÓN }\end{array}$ & \multicolumn{2}{c|}{$\begin{array}{c}\text { ATENCIÓN EN LA } \\
\text { CAJA }\end{array}$} \\
\hline \multirow{2}{*}{ INDICADOR } & CRITERIO & ANTES & DESPUÉS \\
\hline \multirow{4}{*}{ Nivel de satisfacción en la caja } & Excelente & $0 \%$ & $0 \%$ \\
\cline { 2 - 4 } & Bueno & $54 \%$ & $83 \%$ \\
\cline { 2 - 4 } & Regular & $43 \%$ & $17 \%$ \\
\cline { 2 - 4 } & Malo & $3 \%$ & $0 \%$ \\
\cline { 2 - 4 } & Muy Malo & $0 \%$ & $0 \%$ \\
\hline
\end{tabular}

Elaboración propia

El nivel de satisfacción del cliente en relación con rapidez en la atención pasó de un nivel bueno de $36 \%$ a $80 \%$.

TABLA 8

NIVEL DE SATISFACCIÓN DEL CLIENTE EN RELACION A LA RAPIDEZ EN LA ATENCIÓN

\begin{tabular}{|c|c|c|c|c|}
\hline $\begin{array}{c}\text { DIMENSI } \begin{array}{c}\text { ATENCIÓN AL } \\
\text { ONN }\end{array} \\
\text { CLIENTE }\end{array}$ & $\begin{array}{c}\text { SUB } \\
\text { DIMENSIÓN }\end{array}$ & \multicolumn{2}{c|}{$\begin{array}{c}\text { RAPIDEZ EN } \\
\text { ATENCIÓN }\end{array}$} \\
\hline \multirow{2}{*}{ INDICADOR } & CRITERIO & ANTES & DESPUÉS \\
\hline \multirow{4}{*}{$\begin{array}{c}\text { Nivel de satisfacción en la rapidez } \\
\text { de la atención }\end{array}$} & Excelente & $0 \%$ & $0 \%$ \\
\cline { 2 - 5 } & Bueno & $36 \%$ & $80 \%$ \\
\cline { 2 - 5 } & Regular & $57 \%$ & $20 \%$ \\
\cline { 2 - 5 } & Malo & $7 \%$ & $0 \%$ \\
\cline { 2 - 5 } & Muy Malo & $0 \%$ & $0 \%$ \\
\hline
\end{tabular}

Elaboración propia

El nivel de satisfacción del cliente en relación con la agilidad en la solución de un problema pasó de un nivel bueno de $23 \%$ a $77 \%$.

TABLA 9

NIVEL DE SATISFACCIÓN DEL CLIENTE EN RELACION A LA AGILIDAD EN LA SOLUCIÓN DE UN PROBLEMA

\begin{tabular}{|c|c|c|c|c|}
\hline \multirow{2}{*}{$\begin{array}{c}\text { DIMEN } \\
\text { SIÓN }\end{array}$} & $\begin{array}{c}\text { ATENCIÓN AL } \\
\text { CLIENTE }\end{array}$ & \multicolumn{2}{|c|}{$\begin{array}{c}\text { SUB } \\
\text { DIMENSIÓN }\end{array}$} & \multicolumn{2}{c|}{$\begin{array}{c}\text { AGILIDAD EN LA } \\
\text { SOLUCIÓN DE UN } \\
\text { PROBLEMA }\end{array}$} \\
\hline \multirow{2}{*}{ INDICADOR } & CRITERIO & ANTES & DESPUÉS \\
\hline \multirow{3}{*}{$\begin{array}{c}\text { Nivel de satisfacción en la } \\
\text { agilidad de la solución de un } \\
\text { problema }\end{array}$} & Excelente & $0 \%$ & $0 \%$ \\
\cline { 2 - 4 } \begin{tabular}{c} 
prono \\
\cline { 2 - 4 }
\end{tabular} & Bueno & $23 \%$ & $77 \%$ \\
\cline { 2 - 4 } & Regular & $50 \%$ & $23 \%$ \\
\cline { 2 - 4 } & Malo & $27 \%$ & $0 \%$ \\
\cline { 2 - 4 } & Muy Malo & $0 \%$ & $0 \%$ \\
\hline
\end{tabular}

Elaboración propia

El nivel de satisfacción del cliente en relación con los productos pasó de un nivel bueno de $37 \%$ a $87 \%$. 
TABLA 10

NIVEL DE SATISFACCIÓN DEL CLIENTE EN RELACION CON LOS PRODUCTOS

\begin{tabular}{|c|c|c|c|c|}
\hline DIMENSIÓN & $\begin{array}{c}\text { ATENCIÓN AL } \\
\text { CLIENTE }\end{array}$ & \multicolumn{2}{l|}{ SUB DIMENSIÓN } & \multicolumn{2}{c|}{ PRODUCTOS } \\
\hline \multirow{2}{*}{ INDICADOR } & CRITERIO & ANTES & DESPUÉS \\
\hline \multirow{4}{*}{$\begin{array}{c}\text { Nivel de satisfacción sobre los } \\
\text { productos }\end{array}$} & Excelente & $0 \%$ & $0 \%$ \\
\cline { 2 - 4 } & Bueno & $37 \%$ & $87 \%$ \\
\cline { 2 - 4 } & Regular & $23 \%$ & $13 \%$ \\
\cline { 2 - 4 } & Malo & $40 \%$ & $0 \%$ \\
\cline { 2 - 4 } & Muy Malo & $0 \%$ & $0 \%$ \\
\hline
\end{tabular}

Elaboración propia

\section{PRODUCTO}

Para la evaluacion de la satisfacción lo conformaron los clientes que realizaron compras en el primer trimestre del año 2020, cuya muestra fue de 30 clientes.

El nivel de satisfacción del cliente en relación con la variedad de productos pasó de un nivel bueno de $40 \%$ a $73 \%$.

TABLA 11

NIVEL DE SATISFACCIÓN DEL CLIENTE EN RELACION A LA VARIEDAD DE PRODUCTOS

\begin{tabular}{|c|c|c|c|c|}
\hline DIMENSIÓN & \multirow{2}{*}{ PRODUCTOS } & $\begin{array}{c}\text { SUB } \\
\text { DIMENSIÓN }\end{array}$ & $\begin{array}{c}\text { VARIEDAD DE } \\
\text { PRODUCTOS }\end{array}$ \\
\hline \multirow{2}{*}{ INDICADOR } & CRITERIO & ANTES & DESPUÉS \\
\hline \multirow{3}{*}{$\begin{array}{c}\text { Nivel de satisfacción en el } \\
\text { trato amable }\end{array}$} & Excelente & $0 \%$ & $0 \%$ \\
\cline { 2 - 4 } & Bueno & $40 \%$ & $73 \%$ \\
\cline { 2 - 4 } & Regular & $40 \%$ & $23 \%$ \\
\cline { 2 - 4 } & Malo & $20 \%$ & $0 \%$ \\
\cline { 2 - 4 } & Muy Malo & $0 \%$ & $0 \%$ \\
\hline & Elaboración propia & \\
\hline
\end{tabular}

El nivel de satisfacción del cliente en relación con los productos por mala calidad pasó de un nivel nunca de $57 \%$ a $97 \%$.

TABLA 12

NIVEL DE SATISFACCIÓN DEL CLIENTE EN RELACION A LA DEVOLUCIÓN POR MALA CALIDAD

\begin{tabular}{|c|c|c|c|c|}
\hline $\begin{array}{c}\text { DIMENSI } \\
\text { ÓN }\end{array}$ & $\begin{array}{c}\text { RODUC } \\
\text { TO }\end{array}$ & $\begin{array}{c}\text { SUB } \\
\text { DIMENSIÓN }\end{array}$ & \multicolumn{2}{|c|}{$\begin{array}{l}\text { PRODUCTOS POR MALA } \\
\text { CALIDAD }\end{array}$} \\
\hline $\begin{array}{l}\text { INDICAD } \\
\text { OR }\end{array}$ & & CRITERIO & ANTES & DESPUÉS \\
\hline \multirow{5}{*}{$\begin{array}{l}\text { Nivel de } \\
\text { devolución } \\
\text { de } \\
\text { productos } \\
\text { por mala } \\
\text { calidad }\end{array}$} & & Nunca & $57 \%$ & $97 \%$ \\
\hline & & $\begin{array}{c}\text { Muy pocas } \\
\text { veces }\end{array}$ & $30 \%$ & $3 \%$ \\
\hline & & Algunas veces & $13 \%$ & $0 \%$ \\
\hline & & Casi siempre & $0 \%$ & $0 \%$ \\
\hline & & Siempre & $0 \%$ & $0 \%$ \\
\hline
\end{tabular}

El nivel de satisfacción del cliente en relación con el precio pasó de un nivel bueno de $34 \%$ a $70 \%$.

TABLA 13

NIVEL DE SATISFACCIÓN DEL CLIENTE EN RELACION AL PRECIO

\begin{tabular}{|c|c|c|c|c|}
\hline DIMENSIÓN & PRODUCTO & SUB DIMENSIÓN & \multicolumn{2}{|c|}{ PRECIO } \\
\hline \multirow{2}{*}{ INDICADOR } & CRITERIO & ANTES & DESPUÉS \\
\hline \multirow{3}{*}{$\begin{array}{c}\text { Nivel de satisfacción en la } \\
\text { variedad de productos }\end{array}$} & Excelente & $0 \%$ & $0 \%$ \\
\cline { 2 - 4 } & Bueno & $34 \%$ & $70 \%$ \\
\cline { 2 - 4 } & Regular & $53 \%$ & $30 \%$ \\
\cline { 2 - 4 } & Malo & $13 \%$ & $0 \%$ \\
\cline { 2 - 4 } & Muy Malo & $0 \%$ & $0 \%$ \\
\hline
\end{tabular}

\section{ELEMENTOS TANGIBLES}

Para la evaluacion de la satisfacción lo conformaron los clientes que realizaron compras en el primer trimestre del año 2020, cuya muestra fue de 385 clientes.

El nivel de satisfacción del cliente en relación con las instalaciones visualmente atractivas pasó de un nivel bueno de $90 \%$ a $93 \%$.

TABLA 14

NIVEL DE SATISFACCIÓN DEL CLIENTE EN RELACION CON LAS INSTALACIONES VISUALMENTE ATRACTIVAS

\begin{tabular}{|c|c|c|c|c|}
\hline \multirow{2}{*}{ DIMENSIÓN } & $\begin{array}{c}\text { ELEMENTOS } \\
\text { TANGIBLES }\end{array}$ & $\begin{array}{c}\text { SUB } \\
\text { DIMENS } \\
\text { IÓN }\end{array}$ & \multicolumn{2}{c|}{$\begin{array}{c}\text { LAS INSTALACIONES } \\
\text { SON VISUALMENTE } \\
\text { ATRACTIVAS }\end{array}$} \\
\hline \multicolumn{2}{|c|}{ INDICADOR } & CRITERIO & ANTES & DESPUÉS \\
\hline \multirow{3}{*}{$\begin{array}{c}\text { Nivel de satisfacción sobre } \\
\text { la visibilidad de las } \\
\text { instalaciones }\end{array}$} & Excelente & $0 \%$ & $0 \%$ \\
\cline { 2 - 4 } & Bueno & $90 \%$ & $93 \%$ \\
\cline { 2 - 4 } & Regular & $10 \%$ & $7 \%$ \\
\cline { 2 - 4 } & Malo & $0 \%$ & $0 \%$ \\
\cline { 2 - 4 } & Muy Malo & $0 \%$ & $0 \%$ \\
\hline
\end{tabular}

Elaboración propia

El nivel de satisfacción del cliente en relación con el orden y la limpieza de la tienda pasó de un nivel bueno de $80 \%$ a $93 \%$.

TABLA 15

NIVEL DE SATISFACCIÓN DEL CLIENTE EN RELACION CON EL ORDEN Y LIMPIEZA

\begin{tabular}{|c|c|c|c|c|}
\hline $\begin{array}{c}\text { DIMENSI } \\
\text { ÓN } \\
\end{array}$ & $\begin{array}{l}\text { ELEMIENTUS } \\
\text { TANGIBLES }\end{array}$ & \multicolumn{2}{|c|}{$\begin{array}{c}\text { SUB } \\
\text { DIMENSIÓN }\end{array}$} & $\begin{array}{l}\text { ORDEN Y } \\
\text { CIMPIEZA }\end{array}$ \\
\hline \multicolumn{2}{|c|}{ INDICADOR } & CRITERIO & ANTES & DESPUÉS \\
\hline \multirow{5}{*}{\multicolumn{2}{|c|}{$\begin{array}{l}\text { Nivel de orden y limpieza } \\
\text { dentro del local }\end{array}$}} & Excelente & $0 \%$ & $0 \%$ \\
\hline & & Bueno & $80 \%$ & $93 \%$ \\
\hline & & Regular & $17 \%$ & $7 \%$ \\
\hline & & Malo & $3 \%$ & $0 \%$ \\
\hline & & Muy Malo & $0 \%$ & $0 \%$ \\
\hline
\end{tabular}

Elaboración propia

El nivel de satisfacción del cliente en relación con la apariencia pulcra de los trabajadores pasó de un nivel bueno de $17 \%$ a $83 \%$.

TABLA 16

NIVEL DE SATISFACCIÓN DEL CLIENTE EN RELACION CON LA APARIENCIA PULCRA

\begin{tabular}{|c|c|c|c|c|}
\hline $\begin{array}{c}\text { DIMENSI } \\
\text { ÓN }\end{array}$ & $\begin{array}{c}\text { ELEMENTOS } \\
\text { TANGIBLES }\end{array}$ & \multicolumn{2}{c|}{$\begin{array}{c}\text { SUB } \\
\text { DIMENSIÓN }\end{array}$} & \multicolumn{2}{c|}{$\begin{array}{c}\text { APARIENCIA } \\
\text { PULCRA }\end{array}$} \\
\hline \multicolumn{2}{|c|}{ INDICADOR } & CRITERIO & ANTES & DESPUÉS \\
\hline \multirow{4}{*}{$\begin{array}{c}\text { Nivel de orden y limpieza dentro } \\
\text { del local }\end{array}$} & Excelente & $0 \%$ & $0 \%$ \\
\cline { 2 - 5 } & Bueno & $17 \%$ & $83 \%$ \\
\cline { 2 - 5 } & Regular & $53 \%$ & $17 \%$ \\
\cline { 2 - 4 } & Malo & $30 \%$ & $0 \%$ \\
\cline { 2 - 5 } & Muy Malo & $0 \%$ & $0 \%$ \\
\hline
\end{tabular}

Elaboración propia 


\section{CONCLUSIONES}

La empresa Inversiones Pinto S.A.C. no lleva un adecuado manejo de sus procesos ni de la información que se maneja en estos, todas las actividades se realizan empíricamente, ya que no cuenta con un manual de procesos. Además, no tienen definidos sus objetivos, por lo tanto, no lleva una planificación estratégica de lo que quiere lograr.

El diseño propuesto se basa en procesos, lo que permite una estructura plana y reducción de la jerarquización para un mejor control de las actividades que conforman los procesos; disminuyendo los tiempos y costos internos innecesarios, mediante la eliminación de actividades que no generan valor. Factores que ayudarán a la empresa a mejorar la calidad de servicio que ofrece. Para implementar el diseño organizacional propuesto, el equipo de proyecto trabajó de forma conjunta con los trabajadores y gerente de la empresa, dándoles a conocer los cambios que implican la nueva estructura, asimismo, la importancia del proyecto y cuál es su función dentro de este.

A partir de la implementación del nuevo diseño organizacional se obtuvo que la experiencia de compra de los clientes subió a la escala de muy bueno, teniendo que en cuenta que con la gestión anterior el mismo aspecto estaba en una escala regular.

En el plano económico, la propuesta de organización por procesos tuvo una influencia positiva, pues se logró aumentar las ventas y el resultado neto en los meses de abril a junio del 2018 , de $1.79 \%$ a $2.9 \%$ respectivamente. Lo mismo sucedió, en el plano ambiental, a través de plan de gestión ambiental se logró concientizar y fomentar la reutilización y separación de los distintos desechos que genera la empresa. Con ello se logró reducir en un $42 \%$ el uso de papel principalmente. Así mismo, se redujo también el uso de agua y energía eléctrica. Respecto al factor social, se logró que la zona, poco a poco, vaya convirtiéndose en un referente para el sector, a nivel local. Lo que demuestra la viabilidad de la propuesta en los tres sentidos.

\section{REFERENCIAS}

[1] Gestión. (2019). ¿Cree que el mercado automotriz crezca 3\% este año?. Recuperado de: https://gestion.pe/opinion/pregunta-de-hoy/cree-mercadoautomotriz-crezca-3-ano-258532-noticia/?ref=gesr

[2] Gestión. (2018). Mercado automotriz de Latinoamérica creció 7.1\% en el 2018, ¿cómo le fue a Perú? Recuperado de: https://gestion.pe/economia/mercados/mercado-automotriz-latinoamericacrecio-7-1-2018-le-peru-257027-noticia/?ref=gesr

[3] Sector automotriz retrocedió $8 \%$ en 2018. (08 de enero de 2018). El Economista América. Recuperado de: https://www.eleconomistaamerica.pe/economia-eAmperu/noticias/9621828/01/19/Sector-automotriz-retrocedio-8-en-el2018.html.

[4] La República. (2020). AAP: Sector automotor creció 2,4\% durante el año pasado. Recuperado de: https://larepublica.pe/economia/2020/01/14/aapsector-automotor-crecio-24-durante-el-anopasado/\#: : :text=Con $\% 20$ un $\% 20$ crecimiento $\% 20 \mathrm{del} \% 202$, mientras $\% 20 \mathrm{qu}$ e\%20M\%C3\%A9xico\%20y\%20Chile

[5] Pérez, Y. (2016). La mejora continua de los procesos en una organización fortalecida mediante el uso de herramientas de apoyo a la toma de decisiones. Revista Empresarial, (37), p. 9-19. Recuperado de: file:///G:/respaldo20.11.19/Downloads/Dialnet-

LaMejoraContinuaDeLosProcesosEnUnaOrganizacionFort-5580335.pdf

[6] Beltrán et al.(2002). GUÍA PARA UNA GESTIÓN BASADA EN PROCESOS. Sevilla, España: Instituto Andaluz de Tecnología.
[7] Maldonado, J. (2018). Gestión por procesos. Málaga, España: Universidad de Málaga.

[8] Bravo, J. (2009). Gestión de procesos. Santiago, Chile: Evolución S.A.

[9] Tschohl, J. (2014).Servicio al Cliente. Estados Unidos de América: Service Quality Institute.

[10] Tigani, D. (2006). Excelencia en Servicio. Argentina: Liderazgo 21.

[11] Rosales, L. (2010). Componentes esenciales del trabajo en equipo y el servicio al cliente interno y externo. Bibliotecas, 28(2). Recuperado de: https://www.revistas.una.ac.cr/index.php/bibliotecas/article/view/383

[12] Berna, M. (2015). Gestión por procesos y mejora continua, puntos clave para la satisfacción del cliente. Bogotá, Colombia. Recuperado de: https://pdfs.semanticscholar.org/aafa/c592b8dd3fb7cd2c660995638170fb 90484f.pdf

[13] Lira, M. (2009). ¿Cómo puedo mejorar el servicio al cliente?. México: Cámara Nacional de la Industria.

[14] Kotler, P., Keller, K. (2012). Dirección de marketing. México: Pearson Education.

[15] Restrepo et al. (2006). Enfoque estratégico del servicio al cliente. Scientia et Technica Año XII, 32, p. 289-294. Recuperado de: https://www.redalyc.org/pdf/849/84911652051.pdf

[16] Notimerica. (2015). McDonald's, Starbucks, Apple y Uber, empresas que cambian sus negocios. Recuperado de: https://www.notimerica.com/economia/noticia-mcdonalds-starbucksapple-uber-empresas-cambian-negocios-20150512154148.html

[17] Rodriguez, I. Alpuin, D. (2014). La Gestión por Procesos en las Organizaciones. Revista Deloitte. Recuperado de: https://www2.deloitte.com/content/dam/Deloitte/uy/Documents/strategy/G esti\%C3\%B3n\%20por\%20procesos\%20para\%20web.pdf

[18] Hernández, R., Fernández, C. \& Baptista, P. (2010). Metodología De La Investigación. México: McGraw-Hill.

$1^{\text {sh }}$ LACCEI International Multiconference on Entrepreneurship, Innovation and Regional Development - LEIRD 2021: "Ideas to Overcome and Emerge from the Pandemic Crisis", Virtual Edition, December 9-10, 2021. 